\title{
Assistência de enfermagem aos casos leves da COVID-19
}

\author{
Nursing care for mild cases of COVID-19 \\ Cuidado de enfermería para casos leves de COVID-19
}

Thandara Rejane Santos Ferreira Andrade ORCID: https://orcid.org/0000-0002-3385-7727 Universidade Tiradentes, Brasil

E-mail: thandara456@outlook.com

Grayce Ellen Souza Rezende

ORCID: https://orcid.org/0000-0002-6197-5720

Universidade Tiradentes, Brasil

E-mail: grayce-ellen@ hotmail.com

Ianka Heloisa Alencar Santos

ORCID: https://orcid.org/0000-0002-2057-0727

Universidade Tiradentes, Brasil

E-mail: iankaheloisa@outlook.com

Erivelton Cunha Torres

ORCID: https://orcid.org/0000-0002-3866-6305

Universidade Tiradentes, Brasil

E-mail: eriveltontorres18@gmail.com

Sávio Menezes Farias

ORCID: https://orcid.org/0000-0002-1727-9971

Universidade Tiradentes, Brasil

E-mail: savio.menezes@ souunit.com.br

Yasmim Anayr Costa Ferrari

ORCID: https://orcid.org/0000-0003-1766-341X

Universidade Tiradentes, Brasil

E-mail: yasmimanayr@hotmail.com

Anderson Batista Cavalcante

ORCID: https://orcid.org/0000-0002-4168-4379

Universidade Católica de Campinas, Brasil

E-mail: meiro1976@hotmail.com

Rebecca Maria Oliveira de Góis

ORCID: https://orcid.org/0000-0002-3935-5904

Universidade Estadual de Feira de Santana, Brasil

E-mail: rebecca.gois@hotmail.com

Carla Viviane Freitas de Jesus

ORCID: https://orcid.org/0000-0002-7775-6610 Universidade Tiradentes, Brasil

E-mail: carlavfj@gmail.com

Manuela de Carvalho Vieira Martins

ORCID: https://orcid.org/0000-0003-1222-5955 Universidade Tiradentes, Brasil

E-mail: manuela.cvm@hotmail.com

\section{Resumo}

Objetivou-se identificar os possíveis diagnósticos e intervenções de enfermagem em pacientes acometidos pela forma leve da COVID-19 utilizando a taxonomia CIPE 2019. Trata-se de uma revisão integrativa da literatura, realizada por meio de levantamento bibliográfico a partir de dados secundários. O presente estudo analisa e sintetiza um compilado de evidências científicas relacionadas a questão norteadora e permitem a obtenção de conclusões integrais sobre o problema de pesquisa. Foram selecionados nove artigos. Diante da leitura dos estudos selecionados, obteve-se a análise dos sinais e sintomas mais frequentes entre os pacientes adultos com a COVID-19. Observou-se a prevalência de condições clínicas com acometimento sistêmico, sendo elencados febre, fadiga, mialgia, e acometimento respiratório, a exemplo da tosse não produtiva, dispneia, hemoptise. Além destes, foram observadas manifestações relacionadas ao sistema nervoso, como cefaleia, anosmia, ageusia, assim como os presentes no sistema gastrintestinal, tendo em vista, diarreia, náuseas e vômitos. A partir das manifestações mais prevalentes foram elencados Diagnósticos e Intervenções de Enfermagem. O presente estudo possui relevância por elucidar quanto aos Diagnósticos de Enfermagem e as condutas do enfermeiro diante dos casos leves de COVID-19, com a finalidade de estabelecer a sistematização do cuidado de forma satisfatória, perante os eventos relacionados a essa afecção.

Palavras-chave: Infecções por coronavírus; Sinais e sintomas; Diagnósticos de enfermagem. 


\begin{abstract}
The objective was to identify the possible diagnoses and nursing interventions in patients affected by the mild form of COVID-19 using the CIPE 2019 taxonomy. This is an integrative literature review, carried out by means of a bibliographic survey based on secondary data. The present study analyzes and synthesizes a compilation of scientific evidence related to the guiding question and allows for obtaining full conclusions about the research problem. Nine articles were selected. When reading the selected studies, an analysis of the most frequent signs and symptoms was obtained among adult patients with COVID-19. The prevalence of clinical conditions with systemic involvement was observed, including fever, fatigue, myalgia, and respiratory impairment, such as non-productive cough, dyspnoea, hemoptysis. In addition to these, manifestations related to the nervous system were observed, such as headache, anosmia, ageusia, as well as those present in the gastrointestinal system, in view of diarrhea, nausea and vomiting. From the most prevalent manifestations, Nursing Diagnoses and Interventions were listed. The present study has relevance for elucidating the Nursing Diagnoses and the nurses' conduct in the face of mild cases of COVID-19, in order to establish the systematization of care in a satisfactory manner, in the face of events related to this condition.
\end{abstract}

Keywords: Coronavirus infections; Signs and symptoms; Nursing diagnosis.

\title{
Resumen
}

El objetivo de este estudio fue identificar posibles diagnósticos e intervenciones de enfermería en pacientes afectados por la forma leve de COVID-19 utilizando la taxonomía CIPE 2019. Se trata de una revisión integradora de la literatura, realizada mediante una encuesta bibliográfica basada en datos secundarios. El presente estudio analiza y sintetiza una recopilación de evidencia científica relacionada con la pregunta orientadora y permite obtener conclusiones completas sobre el problema de investigación. Se seleccionaron nueve artículos. Al leer los estudios seleccionados, se obtuvo un análisis de los signos y síntomas más frecuentes entre pacientes adultos con COVID-19. Se observó la prevalencia de cuadros clínicos con afectación sistémica, como fiebre, fatiga, mialgias e insuficiencia respiratoria, como tos no productiva, disnea, hemoptisis. Además de estos, se observaron manifestaciones relacionadas con el sistema nervioso, como dolor de cabeza, anosmia, ageusia, así como las presentes en el sistema gastrointestinal, en vista de diarreas, náuseas y vómitos. De las manifestaciones más prevalentes se enumeraron los Diagnósticos e Intervenciones de Enfermería. El presente estudio tiene relevancia para dilucidar los Diagnósticos de Enfermería y la conducta de los enfermeros ante los casos leves de COVID-19, a fin de establecer la sistematización de la atención de manera satisfactoria, ante eventos relacionados con esta condición.

Palabras clave: Infecciones por coronavírus; Signos y sintomas; Diagnósticos de enfermería.

\section{Introdução}

Em 30 de janeiro de 2020, a Organização Mundial de Saúde (OMS) declarou que o surto da doença do novo coronavírus de 2019 (COVID-19) causada pelo agente etiológico Síndrome Respiratória Aguda Grave do Coronavírus 2 (SARS-CoV-2), se constituiu em uma emergência de saúde pública de repercussão mundial. A COVID-19 ligeiramente disseminou-se por todos os continentes, aumentando exponencialmente o número de infectados e provocando milhares de mortes no globo (Carrillo-Larco \& Altez-Fernandez, 2020). Até setembro de 2020, foram confirmados 31.174 .627 casos da COVID-19 e mais de 900.000 mortes no mundo. Nesse mesmo período, no Brasil, cerca de quatro milhões de casos e 133.119 óbitos foram notificados, representando a nação Sul-Americana com maior número de indivíduos contaminados (Opas, 2020).

A transmissão do SARS-CoV-2 ocorre por meio do contato interpessoal, visto que, as gotículas expelidas através do espirro, tosse e fala de pessoas contaminadas, disseminam-se em situações corriqueiras pelo ambiente, propiciando condições favoráveis para o desenvolvimento da infecção, bem como o contato com objetos ou superfícies contaminadas (Brasil, 2020). A despeito da caracterização incipiente da COVID-19 como enfermidade de acometimento e danos respiratórios, atualmente o vírus tem mostrado ação sistêmica, com repercussões clínicas que variam de leves à graves (Dantas et al., 2020). Um considerável percentual de indivíduos contaminados, recupera-se da enfermidade sem precisar de tratamento hospitalar. Entretanto, uma em cada seis pessoas infectadas ficam gravemente doentes (Opas, 2020).

Os sintomas mais comuns são febre, astenia e tosse não produtiva. Contudo, alguns pacientes podem apresentar também congestão nasal, cefaleia, conjuntivite, dor de garganta, diarreia, anosmia e ageusia. Quanto aos grupos de risco, idosos e portadores de doenças crônicas como, Diabetes Mellitus, Hipertensão Arterial Sistêmica e Imunocomprometidos, apresentam maior propensão para desenvolver as condições graves da enfermidade. Nesse cenário, o surto pandêmico, 
classificado como doença infectocontagiosa, torna-se desafiadora no contexto da assistência à saúde e representa constante ameaça para os enfermeiros e todos os profissionais de saúde (Queiroz et al., 2020).

A equipe de enfermagem possui extrema significância no que tange ao cuidado integral e assistencial dos pacientes em todos os níveis de atenção à saúde. Esses profissionais se encontram desde a admissão até a alta dos pacientes, além de possuírem a característica de ser a classe profissional que está em todo o tempo e em todas as etapas da vida, junto ao paciente. É importante evidenciar o papel do enfermeiro como líder e gerenciador do cuidado, exercendo funções que promovem aos profissionais de enfermagem o destaque no enfrentamento da COVID-19 (Queiroz et al., 2020).

A Sistematização da Assistência de Enfermagem (SAE) é uma ferramenta fundamental para o gerenciamento e prestação de cuidados efetivos, através da organização do cuidado, embasados por conhecimento científico e pensamento crítico. A execução da SAE promove o olhar mais direcionado e holístico ao paciente, por conseguinte gerando uma melhor qualidade na assistência aos mesmos. O Processo de Enfermagem (PE) é utilizado para realizar a sistematização da assistência de enfermagem, sendo constituído por cinco etapas: histórico do paciente, diagnóstico de enfermagem, planejamento, implementação e avaliação. Destaca-se as atividades de identificação dos diagnósticos e elaboração das intervenções, visto que são de extrema pertinência para uma assistência direcionada e eficaz aos pacientes com a infecção da COVID-19 (Dantas et al., 2020).

Diante do exposto, o objetivo do presente estudo é identificar os possíveis diagnósticos e intervenções de enfermagem em pacientes acometidos pela forma leve da COVID-19 utilizando a taxonomia da Classificação Internacional para a Prática de Enfermagem (CIPE) versão 2019.

\section{Metodologia}

Trata-se de uma revisão integrativa da literatura, realizada por meio de levantamento bibliográfico a partir de dados secundários entre os meses de setembro e outubro de 2020. O presente estudo analisa e sintetiza um compilado de evidências científicas relacionadas a questão norteadora e permitem a obtenção de conclusões integrais sobre o problema de pesquisa.

A revisão integrativa $(\mathrm{RI})$ é um método que propicia o conhecimento através do processo sistemático e rigoroso. Dessa forma, a construção da RI deve pautar-se em seis etapas: 1) elaboração da pergunta norteadora; 2) busca e seleção dos estudos primários; 3) extração de dados das evidências; 4) avaliação crítica dos estudos primários incluídos na revisão; 5) síntese dos resultados da revisão e 6) apresentação da revisão (Mendes et al., 2019). Por conseguinte, foi elaborada a pergunta norteadora: Quais diagnósticos e intervenções de enfermagem podem ser elencados diante das manifestações clínicas mais prevalentes para os pacientes adultos vítimas da forma leve da Covid-19?

A coleta dos artigos científicos foi realizada nas seguintes bases de dados: Literatura Latino-americana e do Caribe em Ciências da Saúde (LILACS), Medical Literature Analysis and Retrieval System Online (MEDLINE) e Scientific Electronic Library Online (SCIELO). Foram utilizados os Descritores em Ciências da Saúde (DeCS): Infecções por coronavírus, Sinais e Sintomas, e Diagnósticos de enfermagem combinados pelo operador boleando AND. Os descritores foram combinados aos pares para abranger abusca, culminando em duas associações: "Sinais e Sintomas” AND "Infecções por coronavírus" e "Infecções por coronavírus AND "Diagnóticos de enfermagem”.

Adotou-se os seguintes critérios de inclusão: artigos disponíveis na íntegra nos idiomas espanhol, inglês e português; artigos originais e revisões sistemáticas; publicados entre dezembro de 2019 a outubro de 2020 com rigor metodológico e delineação coesa. Os artigos exclusos foram os que apresentavam duplicidade nas bases de dados e especificidade das manifestações clínicas em crianças, gestantes e idosos. 
Figura 1 - Fluxo das etapas de coleta de dados. Aracaju-SE, Brasil, 2020.

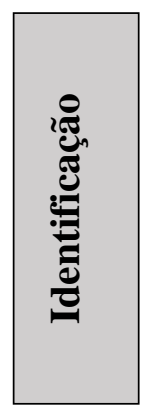

Número de estudos identificados nas bases de dados eletrônicas.

Scielo $(\mathrm{n}=5.889)$ Lilacs $(\mathrm{n}=06)$ Medline $(\mathrm{n}=1.487)$
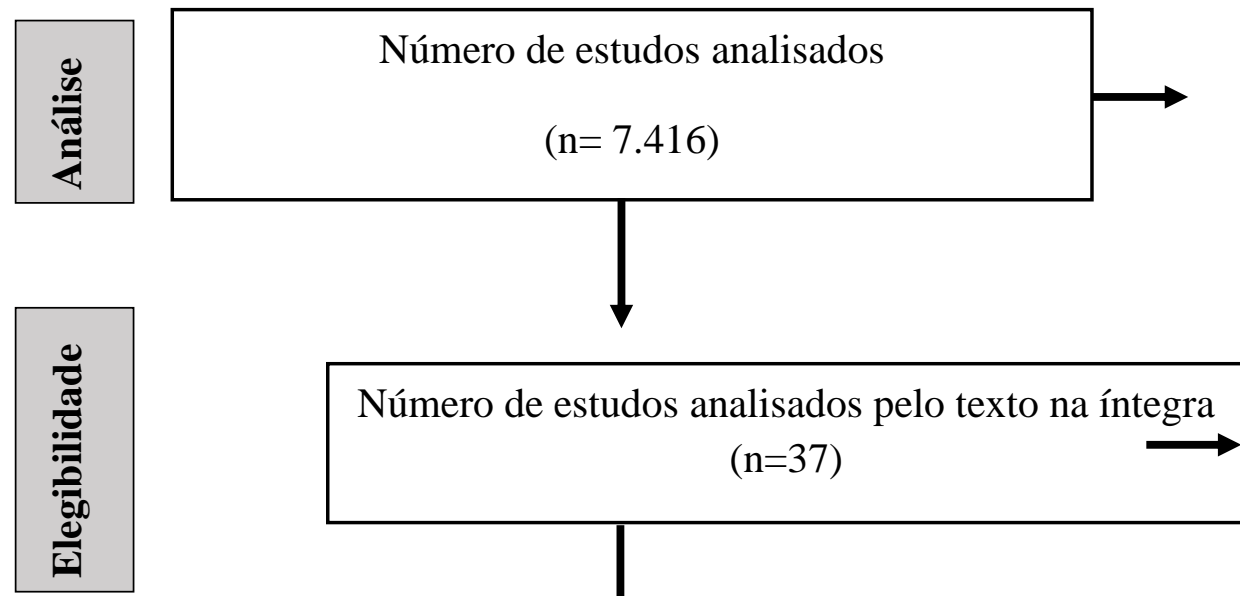

Excluídos por duplicação, pelo título e pelo resumo $(n=7.289)$

Excluídos por conteúdo não pertinente $(\mathrm{n}=28)$

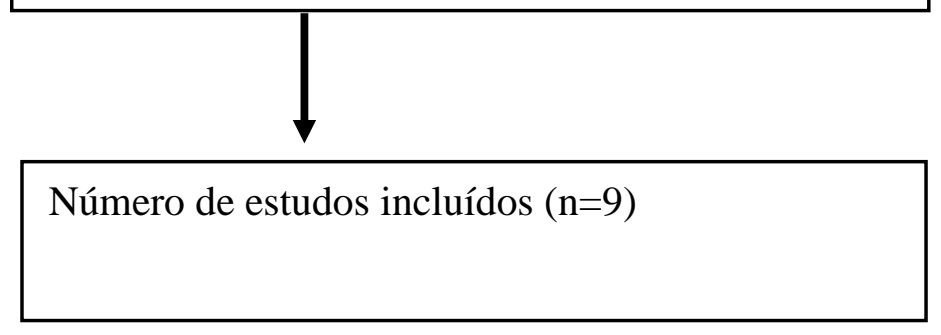

Fonte: Autores.

Conforme a Figura 1, foram descritos o desenvolvimento de seleção dos artigos. Diante das pesquisas por meio dos descritores identificou-se 7.416 artigos. Quando aplicados os critérios de inclusão, resultou-se na seleção de 127 artigos para análise dos títulos e resumos. Em seguida, 37 artigos foram selecionados para leitura na íntegra. A amostra final culminou em 9 artigos que apresentaram critérios de elegibilidade antepostos para RI.

\section{Resultados}

Foram selecionados nove artigos para realização da Revisão Integrativa. Na Tabela 1 localizam-se as informações referentes ao país, período de publicação (mês e ano), título e manifestações clínicas mais prevalentes dos estudos selecionados. 
Tabela 1 - Descrição dos estudos selecionados de acordo com país, período de publicação (mês/ano), título e manifestações clínicas mais prevalentes. Aracaju-SE, Brasil, 2020.

\begin{tabular}{|c|c|c|c|}
\hline País & Período de publicação & Título do artigo & $\begin{array}{l}\text { Manifestações } \\
\text { Clínicas }\end{array}$ \\
\hline China & Fevereiro/2020 & $\begin{array}{l}\text { Clinical characteristics } \\
\text { of hospitalized patients } \\
\text { with SARS-CoV-2 } \\
\text { infections: A single arm } \\
\text { meta-analysis. }\end{array}$ & $\begin{array}{c}\text { Febre } \\
\text { Tosse } \\
\text { Dores musculares } \\
\text { Fadiga }\end{array}$ \\
\hline Colômbia & Março/2020 & $\begin{array}{c}\text { Clinical, laboratory and } \\
\text { imaging features of } \\
\text { COVID-19: A } \\
\text { systematic review and } \\
\text { meta-analysis. }\end{array}$ & $\begin{array}{c}\text { Febre } \\
\text { Tosse } \\
\text { Dispneia }\end{array}$ \\
\hline Colombia & Abril/2020 & $\begin{array}{c}\text { SARS-CoV-2 / COVID- } \\
\text { 19: el vírus, la } \\
\text { enfermedad y la } \\
\text { pandemia. }\end{array}$ & $\begin{array}{l}\text { Febre } \\
\text { Dispneia } \\
\text { Tosse } \\
\text { Fadiga } \\
\text { Mialgia }\end{array}$ \\
\hline Brasil & Abril/2020 & $\begin{array}{l}\text { Padrões funcionais de } \\
\text { saúde em adultos com } \\
\text { COVID-19 na terapia } \\
\text { intensiva: } \\
\text { Fundamentando } \\
\text { diagnósticos de } \\
\text { enfermagem. }\end{array}$ & $\begin{array}{c}\text { Febre } \\
\text { Diarreia } \\
\text { Náuseas } \\
\text { Vômitos }\end{array}$ \\
\hline Japão & Junho/2020 & $\begin{array}{l}\text { Trends in clinical } \\
\text { features of novel } \\
\text { coronavirus disease } \\
\text { (COVID-19):A } \\
\text { systematic review and } \\
\text { meta-analysis of studies } \\
\text { published from } \\
\text { December } 2019 \text { to } \\
\text { February } 2020\end{array}$ & $\begin{array}{l}\text { Febre,Tosse, } \\
\text { Dispneia, } \\
\text { Mialgia } \\
\text { Fadiga, } \\
\text { Cefaleia, } \\
\text { Dor de garganta e } \\
\text { Diarreia }\end{array}$ \\
\hline Brasil & Junho/2020 & $\begin{array}{c}\text { Diagnósticos de } \\
\text { enfermagem segundo a } \\
\text { taxonomia da NANDA } \\
\text { internacional para } \\
\text { sistematização da } \\
\text { assistência de } \\
\text { enfermagem a COVID- } \\
19 . \\
\end{array}$ & $\begin{array}{c}\text { Febre } \\
\text { Tosse } \\
\text { Dispneia } \\
\text { Mialgia } \\
\text { Alterações gastrointestinais } \\
\text { Cefaleia }\end{array}$ \\
\hline Inglaterra & Junho/2020 & $\begin{array}{c}\text { The prevalence of } \\
\text { symptoms in } 24,410 \\
\text { adults infected by the } \\
\text { novel coronavirus } \\
\text { (SARS-CoV-2; COVID- } \\
\text { 19): A systematic } \\
\text { review and meta- } \\
\text { analysis of } 148 \text { studies } \\
\text { from } 9 \text { countries. }\end{array}$ & $\begin{array}{l}\text { Febre } \\
\text { Tosse } \\
\text { Fadiga }\end{array}$ \\
\hline $\begin{array}{l}\text { Estados } \\
\text { Unidos da } \\
\text { América }\end{array}$ & Julho/2020 & $\begin{array}{l}\text { Correlations of Clinical } \\
\text { and Laboratory } \\
\text { Characteristics of COVID- } \\
\text { 19: A systematic Review } \\
\text { and Meta-Analysis. }\end{array}$ & $\begin{array}{c}\text { Febre } \\
\text { Tosse } \\
\text { Fadiga } \\
\text { Mialgia }\end{array}$ \\
\hline
\end{tabular}




\begin{tabular}{|c|c|c|c|}
\hline China & Agosto/2020 & $\begin{array}{l}\text { Epidemiologic, clinical } \\
\text { and laboratory findings } \\
\text { of COVID-19 in the } \\
\text { current pandemic: } \\
\text { systematic review and } \\
\text { meta-analysis }\end{array}$ & $\begin{array}{c}\text { Febre } \\
\text { Tosse } \\
\text { Fadiga } \\
\text { Anosmia } \\
\text { Augesia } \\
\text { Dispneia } \\
\text { Dor de garganta } \\
\text { Cefaleia }\end{array}$ \\
\hline
\end{tabular}

Fonte: Autores.

Diante da leitura dos artigos selecionados, obteve-se a análise dos sinais e sintomas mais frequentes entre os pacientes adultos com a COVID-19. Observou-se a prevalência de condições clínicas com acometimento sistêmico, sendo elencados febre, fadiga, mialgia (Díaz-Castrillón \& Toro-Montoya, 2020; Ghayda et al., 2020; Sun et al., 2020), e acometimento respiratório, a exemplo da tosse não produtiva, dispneia, hemoptise (Grant et al., 2020; Rodriguez-Morales et al., 2020; Xie et al., 2020). Além destes, foram observadas manifestações relacionados ao sistema nervoso, como a dor de cabeça, anosmia, ageusia, assim como os presentes no sistema gastrintestinal, tendo em vista, diarreia, náuseas e vômitos (Bitencourt et al., 2020; Manabe et al., 2020; Queiroz et al., 2020).

Quadro 1 - Diagnósticos e Intervenções de Enfermagem da CIPE de acordo com os sinais e sintomas mais prevalentes apresentados no paciente adulto com COVID-19. Aracaju-SE, Brasil, 2020.

\begin{tabular}{|c|c|}
\hline Diagnóstico de Enfermagem & Intervenções de Enfermagem \\
\hline Febre & $\begin{array}{l}\text { - Acompanhar curva de temperatura e verificar a } \\
\text { temperatura corporal de } 4 / 4 \mathrm{~h} ; \\
\text { - } \quad \text { Inspecionar a ingestão e a eliminação de líquidos; } \\
\text { - Monitorar reações de desorientação/confusão. }\end{array}$ \\
\hline Fadiga & $\begin{array}{l}\text { - Identificar fatores que desencadeiam a fadiga; } \\
\text { - Planejar a assistência para garantir que o paciente tenha } \\
\text { períodos de repouso; } \\
\text { - Orientar o paciente a não fazer esforços e conversar } \\
\text { sobre as mudanças no estilo de vida impostas pela } \\
\text { fadiga. }\end{array}$ \\
\hline Dor Muscular & $\begin{array}{l}\text { - Avaliar a etiologia e fatores contribuintes da dor. } \\
\text { - Monitorar avaliação abrangente da dor, quanto a } \\
\text { localização, início, frequência e duração; } \\
\text { - Avaliar a eficácia das medidas de controle da dor. }\end{array}$ \\
\hline Tosse & $\begin{array}{l}\text { - } \\
\text { - } \\
\text { - } \\
\text { - Obstimular o paciente a ingesta hídrica; } \\
\end{array}$ \\
\hline Dispnéia & $\begin{array}{l}\text { - Avaliar a frequência, profundidade da respiração e } \\
\text { auscultar sons respiratórios, observando se há presença } \\
\text { de ruídos adventícios; } \\
\text { - Elevar a cabeceira da cama e proporcionar uma posição } \\
\text { confortável e adequada para o paciente; } \\
\text { - Instalar suporte de } \mathrm{O} 2 \text { conforme prescrição médica e }\end{array}$ \\
\hline
\end{tabular}




\begin{tabular}{|c|c|}
\hline & monitorar a SatO2. \\
\hline Dor de cabeça & $\begin{array}{l}\text { - Administrar medicação de efeito analgésico e } \\
\text { acompanhar se houve eficácia do medicamento; } \\
\text { - Avaliar o limiar e característica da dor; } \\
\text { - Manter o ambiente tranquilo. }\end{array}$ \\
\hline Olfato prejudicado & $\begin{array}{l}\text { - Reforçar que a alteração do senso olfativo é } \\
\text { consequência da COVID-19; } \\
\text { - } \begin{array}{l}\text { Estimular o paciente a cheirar substâncias que tenho } \\
\text { um cheiro moderado ou forte, duas vezes ao dia; }\end{array} \\
\text { - Registrar a perda do olfato no prontuário do paciente, a } \\
\text { fim de que os demais profissionais tenham ciência. }\end{array}$ \\
\hline Paladar prejudicado & $\begin{array}{l}\text { - Orientar o paciente que a perda do paladar é um } \\
\text { sintoma da COVID-19; } \\
\text { - Estimular o paciente a alimentasse mesmo sem estar } \\
\text { sentindo o gosto do alimento; } \\
\text { - Inspecionar frequentemente a cavidade oral para } \\
\text { detectar possíveis anormalidades; }\end{array}$ \\
\hline Diarreia & $\begin{array}{l}\text { - Traçar fatores causadores e/ou contribuintes da } \\
\text { diarreia; } \\
\text { - Monitorar as eliminações intestinais, inclusive } \\
\text { frequência, consistência, volume e cor; } \\
\text { - Orientar o acompanhante sobre a importância da dieta } \\
\text { constipante. }\end{array}$ \\
\hline Presença de náuseas & 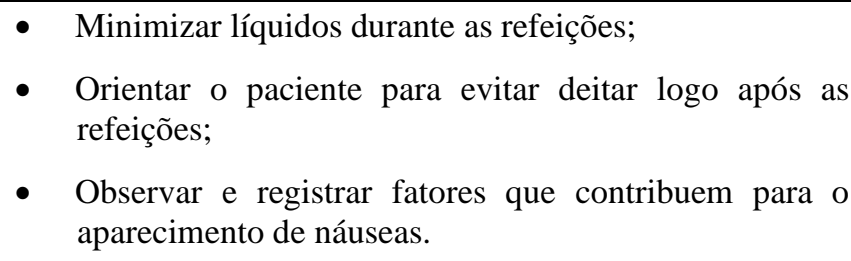 \\
\hline Presença de vômito & $\begin{array}{l}\text { - Manter o paciente com cabeceira elevada e a cabeça } \\
\text { lateralizada; } \\
\text { - Administrar antieméticos e monitorar a frequência dos } \\
\text { episódios de vômitos; } \\
\text { - Avaliar quantidade e coloração do vômito. }\end{array}$ \\
\hline
\end{tabular}

Fonte: Autores.

De acordo com os achados e recursos da CIPE, foram elencados os Diagnósticos de Enfermagem (DE) mais pertinentes à situação clínica dos pacientes adultos com a COVID-19 (Quadro 1). No tocante as intervenções de enfermagem (IE), foram destinadas três intervenções para cada diagnóstico descrito (Quadro 1).

\section{Discussão}

Os diagnósticos e intervenções de enfermagem são etapas que compõem o PE e contribuem na qualidade da assistência prestada aos pacientes. O DE constitui-se a partir da análise, interpretação e agrupamento dos dados coletados na primeira etapa do PE, que resulta na elaboração dos conceitos diagnósticos e representam com exatidão, as respostas da pessoa, família ou coletividade humana em um dado momento do processo saúde e doença. Ainda assim, no Planejamento de 
Enfermagem ocorre a determinação dos resultados que se espera alcançar, e das IE que serão implementadas com o intuito de sistematizar e priorizar os cuidados de acordo com as manifestações clínicas apresentadas e visualizar o paciente de maneira integral (Queiroz et al., 2020).

Nesse contexto, encontra-se a taxonomia CIPE, que promove a padronização dos conceitos diagnósticos que serão utilizados pelo profissional enfermeiro. A CIPE é uma terminologia ampla e complexa, que representa o domínio da prática de enfermagem no âmbito mundial, composta por sete eixos (Foco; Julgamento; Meios; Ação; Tempo; Localização; Cliente) que permitem a construção organizada de conceitos diagnósticos, resultados esperados e intervenções. Dessa forma, para elaboração do DE faz-se obrigatório o uso dos eixos foco e julgamento, exceto quando o termo foco faz parte de um achado clínico. Em contrapartida, para a construção das IE é necessário o eixo ação e uso de termos alvos de outros eixos (Cipe, 2019).

A COVID-19 representa atualmente o maior entrave sanitário no globo e apresenta as mais variadas condições clínicas. A febre compõe um dos sintomas mais esperados nesse agravo, essa elevação da temperatura se dá por meios de falhas no hipotálamo e é ocasionada pela defesa do organismo diante da infecção pelo SARS-CoV-2. Nesse contexto, a hipertermia é a manifestação mais predominante, com incidência maior que 89\% (Sun et al., 2020). Em outra metanálise, observou-se que a frequência de febre foi significativamente mais prevalente em adultos com a idade média de 47,9 anos, e a variável sexo sendo mais significativa no sexo masculino com 69,1\%, sendo avaliada uma amostra de 656 pacientes (Rodriguez-Morales et al., 2020). Portanto, o diagnóstico de enfermagem "Febre" condiz com o contexto dos pacientes com COVID-19, pois trata-se da manifestação mais prevalente apresentada nos pacientes acometidos pela doença. Dessa forma, vale ressaltar alguns cuidados de enfermagem para essa manifestação, dentre elas estão, acompanhar curva de temperatura e verificar a temperatura corporal de 4/4h (Cipe, 2019).

Outrossim, faz-se também presente o diagnóstico de enfermagem "Fadiga", que consiste na redução da força e cansaço contínuo (Cipe, 2019). Destaca-se como um dos sintomas provocado pelo SARS-COV-2 com frequência estimada $41.1 \%$ nos casos confirmados (Manabe et al., 2020). A infecção viral é um dos as mecanismos que provocam a exaustão, causando no individuo infectado a incapacidade muscular e sensação de esgotamento. Com isso, faz-se necessário traçar cuidados de enfermagem como: Identificar fatores que desencadeiam a fadiga; Planejar a assistência para garantir que o paciente tenha períodos de repouso; Orientar o paciente a não fazer esforços e conversar sobre as mudanças no estilo de vida impostas pela fadiga (Cipe, 2019).

O diagnóstico de enfermagem “Dor Muscular" é marcado pela percepção de dor que poder estar associada a diversos fatores, entre eles está presente a infecção (Cipe, 2019). A mialgia é um dos sintomas mais comuns apresentados em pacientes acometidos pela COVID-19, tendo uma incidência de 31,7\% dos casos presentes nos estudos (Ghayda et al., 2020). Diante do DE dor muscular foram elencadas IE como: Avaliar a etiologia e fatores contribuintes da dor; Monitorar avaliação abrangente da dor, quanto a localização, início, frequência e duração (Cipe, 2019).

Outro diagnóstico de enfermagem presente é a "Tosse", que é definida pela saída forçada de ar presente nos pulmões. Pode ser caracterizada como seca ou produtiva, com a mesma frequência, e por vezes acompanhada com hemoptise (DíazCastrillón \& Toro-Montoya, 2020). Tal manifestação clínica apresenta significativa prevalência nos indivíduos acometidos pela COVID-19, com frequência estimada de 62,36\% de todos dos casos da doença (Manabe et al., 2020). Portanto é necessário delinear alguns cuidados de relacionados ao DE tosse, como: Estimular o paciente a ingesta hídrica; Monitorar os sinais vitais; Observar padrão respiratório (Cipe, 2019).

Em relação ao dignóstico de "Dispneia", descrita a partir da dificuldade respiratória sem esforço físico, tendo como principal particularidade a respiração rápida e curta, podendo ser ocasionado por diversos fatores, entre eles alterações no sistema respiratório ou cardiovascular (Cipe, 2019). O DE dispneia é considerado um dos mais prevalentes diante do contexto 
da COVID-19, com frequência de incidência em mais 60\% dos casos, tal sintoma pode surgir a partir do segundo dia após a infecção ou demorar até 17 dias para se manifestar, tendo em vista que quanto mais tardio o aparecimento, mais grave a situação clínica do paciente pode se tornar (Díaz-Castrillón \& Toro-Montoya, 2020). Desta forma, para conduzir um método terapêutico eficaz e humanizado foram elencadas as seguintes IE: Avaliar a frequência e profundidade da respiração e auscultar os sons respiratórios, observando se há presença de ruídos adventícios; Elevar a cabeceira da cama e proporcionar uma posição confortável e adequada para o paciente; e, Instalar oxigenoterapia conforme prescrição médica, bem como monitorar a saturação do indivíduo (Cipe, 2019).

A cefaleia é uma das manifestações clínicas mais comuns na infecção por SARS-CoV-2, possuindo incidência de $15,49 \%$. Ademais, tal sintomatologia ocasiona desordens na qualidade vida dos indivíduos, afetando negativamente os mesmos (Xie et al., 2020). Assim, o DE “Dor de cabeça” é de suma importância para a triagem e identificação dos pacientes, além de proporcionar o cuidado direcionado para tal sintoma. Portanto, algumas intervenções de enfermagem para a dor de cabeça se definem como: Avaliar o limiar e características da dor; Manter o ambiente tranquilo, a fim de incentivar ao repouso; Administrar analgésicos e acompanhar se houve eficácia do medicamento (Xie et al., 2020).

A anosmia que se caracteriza por ausência do olfato, e a augesia como a ausência do paladar são distúrbios com elevada frequência na COVID-19, além de serem as primeiras manifestações clínicas apresentadas na maior parte dos pacientes. Os mecanismos virais no olfato e paladar não são totalmente precisos, contudo, é possível a ação do vírus no epitélio olfativo por meio da Enzima Conversora de Angiotensina 2 (ECA2) que atua ligando-se ao vírus. Ademais, em relação ao paladar foi visualizada semelhança entre o SARS-CoV e o SARS-CoV-2, pois ambos afetam as células epiteliais dos ductos das glândulas salivares (Díaz-Castrillón \& Toro-Montoya, 2020).

Sendo assim, o diagnóstico de enfermagem de "Olfato prejudicado" e "Paladar prejudicado" enquadram-se no contexto da COVID-19, pois afeta os sensos olfativos e gustativos dos pacientes ocasionando repercussões negativas para a vida dos mesmos (Díaz-Castrillón \& Toro-Montoya, 2020). Dessa forma, destacam-se entre os cuidados de enfermagem para tais manifestações: Reforçar que a alteração do senso olfativo é consequência da COVID-19; Estimular o paciente a cheirar substâncias que tenham um cheiro moderado ou forte, duas vezes ao dia; Registrar a perda do olfato no prontuário do paciente, a fim de que os demais profissionais tenham ciência. Orientar o paciente que a perda do paladar é um sintoma da COVID-19; Estimular o paciente a alimentasse mesmo sem estar sentindo o gosto do alimento; Inspecionar frequentemente a cavidade oral para detectar possíveis anormalidades (Cipe, 2019).

As aparições de sintomas gastrointestinais, como náuseas, vômitos e diarreia, apresentam-se inicialmente entre $10 \%$ a $20 \%$ dos pacientes infectados pelo SARS-CoV. O vírus se liga aos receptores da enzima conversora de angiotensina 2 (ACE2) do trato gastrointestinal, que permite a entrada do agente etiológico nas células epiteliais, causando disfunções entéricas significativas (Bitencourt et al., 2020; Díaz-Castrillón \& Toro-Montoya, 2020; Manabe et al., 2020; Queiroz et al., 2020). Tais condições contribuem para perda de eletrólitos fundamentais na homeostase do organismo. Foram caracterizados os diagnósticos "Diarreia", "Presença de náuseas" e "Presença de vômitos", bem como as respectivas intervenções para a avaliação criteriosa do desenvolvimento das manifestações e método terapêutico adequado (Cipe, 2019).

Em suma, a compreensão da COVID-19 é um caminho em evolução contínua, regado de incertezas. Dessa forma, desponta a necessidade de uma observação holística e pormenorizada por parte do profissional enfermeiro, tendo em vista que juntamente com a equipe de enfermagem protagonizam assistência beira leito e promovem o seguimento terapêutico dos pacientes. Nesse contexto, torna-se imprescindível a implementação do PE com ênfase nos DE e IE, a fim de proporcionar melhores condições de cuidado.

Como fatores limitantes para construção do presente estudo, destaca-se a reduzida quantidade de pesquisas publicadas acerca dos DE e IE, assim como restrição de informações sobre a relação fisiopatológica do novo coronavirus e os 
sintomas clínicos que se manifestam nesta afecção.

\section{Considerações Finais}

O presente estudo possui relevância por elucidar quanto aos DE e as condutas do enfermeiro perante os casos leves de COVID-19, com a finalidade de estabelecer a sistematização do cuidado de forma satisfatória, perante os eventos relacionados a essa afecção.

De acordo com análise das evidências científicas selecionadas foram destacadas as manifestações clínicas mais prevalentes nos casos leves de COVID-19, bem como traçados diagnósticos e intervenções de enfermagem a partir da taxonomia CIPE.

Apesar da identificação de 11 DE e suas respectivas intervenções, salienta-se que o processo de enfermagem deve ser realizado de maneira holística e cuidadosa pelo enfermeiro. Assim, é imprescindível que o profissional realize uma investigação (anamnese e exame físico) criteriosa de seus pacientes para que possa identificar os DE e IE de forma correta, pensando na individualidade de cada cliente.

Recomenda-se portanto, para trabalhos futuros o desenvolvimento e construção de novas investigações para fomentar uma necessidade expressiva de melhorar a assistência direta ao paciente, através da implementação do processo de enfermagem. Dessa forma, contemplando a atenção integral e singular para cada indivíduo.

\section{Referências}

Bitencourt, G. R., Elias, J., Barbosa, S., Marx, C., Taets, C., Ceccon, L., Coutinho, R. P., Glauco, G., \& Carelli, D. C. (2020). Padrões funcionais de saúde em adultos com covid-19 na terapia intensiva : fundamentando diagnósticos de enfermagem. International Journal of Development Research, 10(06), 3654036544 .

Brasil. (2020). Ministério da Saúde. Protocolo de Manejo Clínico do Coronavírus (Covid-19) na Atenção Primária à Saúde. Brasília: Ministério da Saúde.

Carrillo-Larco, R. M., \& Altez-Fernandez, C. (2020). Anosmia and dysgeusia in COVID-19: A systematic review. Wellcome Open Research, 5(94). https://doi.org/10.12688/wellcomeopenres.15917.1

Conselho Internacional de Enfermagem. (2020). Classificação Internacional para Prática da Enfermagem: CIPE®. Versão 2019/2020. Porto Alegre: Artmed, 2020 .

Dantas, T. P., Alexandro, C., Teixeira, V. R., Roriston, R., Isabel, M., Rafael, L., Sampaio, L., \& Pinheiro, W. R. (2020). Diagnósticos de enfermagem para pacientes com COVID-19. Journal Health NPEPS, 5(1), 396-416. https://doi.org/http://dx.doi.org/10.30681/252610104575

Díaz-Castrillón, F. J., \& Toro-Montoya, A. I. (2020). SARS-CoV-2/COVID-19: el virus, la enfermedad y la pandemia. Medicina y Laboratorio, 24(3), 183205. https://doi.org/10.36384/01232576.268

Ghayda, R. A., Lee, J., Lee, J. Y., Kim, D. K., Lee, K. H., Hong, S. H., Han, Y. J., Kim, J. S., Yang, J. W., Kronbichler, A., Smith, L., Koyanagi, A., Jacob, L., \& Shin, J. Il. (2020). Correlations of Clinical and Laboratory Characteristics of COVID-19: A Systematic Review and Meta-Analysis. International Journal of Environmental Research and Public Health, 17(14), 5026. https://doi.org/10.3390/ijerph17145026

Grant, M. C., Geoghegan, L., Arbyn, M., Mohammed, Z., McGuinness, L., Clarke, E. L., \& Wade, R. G. (2020). The prevalence of symptoms in 24,410 adults infected by the novel coronavirus (SARS-CoV-2; COVID-19): A systematic review and meta-analysis of 148 studies from 9 countries. PLoS ONE, 15(6 June). https://doi.org/10.1371/journal.pone.0234765

Manabe, T., Akatsu, H., Kotani, K., \& Kudo, K. (2020). Trends in clinical features of novel coronavirus disease (COVID-19): A systematic review and metaanalysis of studies. Respiratory Investigation, 58(5), 409-418. https://doi.org/10.1016/j.resinv.2020.05.005

Mendes, K. D. S., Silveira, R. C. de C. P., \& Galvão, C. M. (2019). Uso de gerenciador de referências bibliográficas na seleção dos estudos primários em revisão integrativa. In Texto \& Contexto - Enfermagem (Vol. 28). scielo.

Opas. (2020). Organização Pan-Americana da Saúde. Folha informativa - COVID-19 (doença causada pelo novo coronavírus). https://www.paho.org/bra/index.php?option=com_content\&view=article\&id=61 01:covid19\&Itemid=875

Queiroz, A. G. S., De Souza, R. Z., Sottocornola, S. F., Barbosa, S. J., Pinheiro, F. A., \& Souza, L. P. de. (2020). Diagnósticos de enfermagem segundo a taxonomia da NANDA internacional para sistematização da assistência de enfermagem a COVID-19. Journal of Health \& Biological Sciences, 8(1), 1. https://doi.org/10.12662/2317-3076jhbs.v8i1.3352.p1-6.2020

Rodriguez-Morales, A. J., Cardona-Ospina, J. A., Gutiérrez-Ocampo, E., Villamizar-Peña, R., Holguin-Rivera, Y., Escalera-Antezana, J. P., Alvarado-Arnez, L. E., Bonilla-Aldana, D. K., Franco-Paredes, C., Henao-Martinez, A. F., Paniz-Mondolfi, A., Lagos-Grisales, G. J., Ramírez-Vallejo, E., Suárez, J. A., Zambrano, L. I., Villamil-Gómez, W. E., Balbin-Ramon, G. J., Rabaan, A. A., Harapan, H., Sah, R. (2020). Clinical, laboratory and imaging features of 
Research, Society and Development, v. 10, n. 1, e5310111307, 2021

(CC BY 4.0) | ISSN 2525-3409 | DOI: http://dx.doi.org/10.33448/rsd-v10i1.11307

COVID-19: A systematic review and meta-analysis. Travel Medicine and Infectious Disease, 34, 101623. https://doi.org/10.1016/j.tmaid.2020.101623

Sun, P., Qie, S., Liu, Z., Ren, J., Li, K., \& Xi, J. (2020). Clinical characteristics of hospitalized patients with SARS-CoV-2 infection: A single arm metaanalysis. Journal of Medical Virology, 92(6), 612-617. https://doi.org/10.1002/jmv.25735

Xie, Y., Wang, Z., Liao, H., Marley, G., Wu, D., \& Tang, W. (2020). Epidemiologic, clinical, and laboratory findings of the COVID-19 in the current pandemic: systematic review and meta-analysis. BMC Infectious Diseases, 20(1), 640. https://doi.org/10.1186/s12879-020-05371-2 